\title{
MINIMIZING FUNCTIONALS DEPENDING ON SURFACES AND THEIR CURVATURES: A CLASS OF VARIATIONAL PROBLEMS IN THE SETTING OF GENERALIZED GAUSS GRAPHS
}

\author{
Silvano Delladio
}

\begin{abstract}
We consider a family of variational problems involving generalized Gauss graphs. Roughly, we are interested in the problem of minimizing a functional defined in a class of surfaces constrained to include a given fixed rectifiable set. As a particular case, one has the following example: Given a rectifiable set $M$, find a mass minimizer among all null boundary generalized Gauss graphs of surfaces which include $M$ as a subset. Particular attention is paid to the case of curves in the plane.
\end{abstract}

\section{Introduction.}

The variational problems where the functional to minimize depends on manifolds and involves curvatures are far to be exhaustively investigated. Probably the most important example of functional of this type is Willmore's one, introduced in the sixties (see [23], [24]) and recently reconsidered by Leon Simon who proved a beautiful existence result in the genus one case (see [21], [22]). For an introductory presentation of Willmore problem one can refer to [18].

General theories about minimizing functionals depending on curvatures can be found in [12] and in [5]. In both of these papers the authors spend a lot of work to define a generalized notion of surface endowed with a curvature tensor and to look into its properties. So Hutchinson defines the curvature varifolds while Anzellotti, Serapioni and Tamanini get the notion of generalized Gauss graph.

A comparison between such generalizations can be found in [9]. Further investigations and applications involving them are given, for example, in [2], [4], [10], [13], [14] and [15].

Here we consider a family of variational problems involving generalized Gauss graphs. Roughly, we are interested in the problem of minimizing a 
functional defined in a class of surfaces constrained to include a given fixed rectifiable set. As a particular case, one has the following example:

Given a rectifiable set $M$, find a mass minimizer among all null boundary generalized Gauss graphs of surfaces which include $M$ as a subset.

The Section 5 is devoted to the presentation of the problem and to get the existence. In 6 we prove that a factor of a minimizer has to be itself a minimizer of a suitable "restricted" problem. Finally, particular attention is paid to the case of curves in the plane, which is discussed in 7. The Sections 2 and 3 recall some standard terminology and the needed preliminaries about generalized Gauss graphs. Some basic facts, particularly with regard to the factors of a generalized Gauss graph, are proved in 4 .

\section{Notation.}

For the general terminology we refer to the classical literature about geometric measure theory (see [11], [17] and [20]). Rectifiable currents are the main tools we will work with.

Let $U$ be an open subset of a given euclidean space. Then we shall denote by $\mathcal{D}_{h}(U)$ the set of $h$-dimensional currents in $U$, while $\mathcal{R}_{h}(U)$ and $\mathbf{I}_{h}(U)$ will be the subspace of rectifiable currents and the subspace of integral currents, respectively. According to a completely standard notation, the set of smooth differential $h$-forms having compact support in $U$ will be denoted by $\mathcal{D}^{h}(U)$.

If $T$ is a $h$-dimensional current, then we define

$$
\mathbf{N}(T):=\mathbf{M}(T) \quad \text { in case } h=0
$$

and

$$
\mathbf{N}(T):=\mathbf{M}(T)+\mathbf{M}(\partial T) \quad \text { in case } h \geq 1
$$

where $\mathbf{M}$ is the usual mass of currents.

Throughout this paper, we will deal with a generalized notion of Gauss graph for surfaces of dimension $n$ and codimension $k$. Thus, the ambients where our surfaces and their generalized Gauss graphs are immersed will be respectively the euclidean spaces $\mathbf{R}^{N}$ and $\mathbf{R}^{N} \times S_{1}\left(\Lambda^{k} \mathbf{R}^{N}\right)$, where:

$$
N:=n+k
$$

and $S_{1}$ denotes the set of unit vectors in the argument space (see Definition 3.1 below).

As we shall soon see, it is convenient to introduce a further copy of $\mathbf{R}^{N}$ which has to be kept distinguished from the ambient space. By specifying 
the variable name into the notation, we will denote them by $\mathbf{R}_{x}^{N}$ and $\mathbf{R}_{\tilde{x}}^{N}$ respectively. Let $e_{1}, \ldots, e_{N}$ (resp. $d x^{1}, \ldots, d x^{N}$ ) be the standard basis (resp. dual basis) of $\mathbf{R}_{x}^{N}$ and let $\varepsilon_{1}, \ldots, \varepsilon_{N}$ (resp. $d \tilde{x}^{1}, \ldots, d \tilde{x}^{N}$ ) be the one of $\mathbf{R}_{\tilde{x}}^{N}$. Then we denote by $\tilde{z} \in \mathbf{R}_{\tilde{x}}^{N}$ the image of $z \in \mathbf{R}_{x}^{N}$ through the trivial isomorphism

$$
\mathbf{R}_{x}^{N} \ni e_{j} \rightarrow \tilde{e}_{j}:=\varepsilon_{j} \in \mathbf{R}_{\tilde{x}}^{N}
$$

i.e.,

$$
\tilde{z}=\sum_{j=1}^{N} z^{j} \varepsilon_{j}
$$

if $z=\sum_{j=1}^{N} z^{j} e_{j}$.

Given a couple of positive integers ( $H$ and $h$ such that $H \geq h$ ), we adopt the usual notation for multi-indices sets:

$$
I(H, h):=\left\{\delta=\left(\delta_{1}, \ldots, \delta_{h}\right) \mid 1 \leq \delta_{1}<\delta_{2}<\ldots<\delta_{h} \leq H\right\} .
$$

Also we need to extend this notation to infinite indices:

$$
I(\infty, h):=\cup_{H} I(H, h) \quad \text { and } \quad I(\infty):=\cup_{h} I(\infty, h) .
$$

The short notation for wedge products will be often used. For example:

$$
e_{\alpha}:=e_{\alpha_{1}} \wedge \ldots \wedge e_{\alpha_{n}} \quad \text { or } \quad d x^{\beta}:=d x^{\beta_{1}} \wedge \ldots \wedge d x^{\beta_{k}} .
$$

Note that $\left\{\varepsilon_{\beta}\right\}_{\beta \in I(N, k)}$ is the basis of $\Lambda^{k}\left(\mathbf{R}_{\tilde{x}}^{N}\right)$ naturally induced from $\mathbf{R}_{\tilde{x}}^{N}$, as well as $\left\{d \tilde{x}^{\beta}\right\}_{\beta \in I(N, k)}$ is the standard basis of the dual space of $\Lambda^{k}\left(\mathbf{R}_{\tilde{x}}^{N}\right)$, et cetera.

The letter $y$ will be adopted to denote the variable of $\Lambda^{k} \mathbf{R}_{\tilde{x}}^{N}$ equipped with the basis $\left\{\varepsilon_{\beta}\right\}_{\beta \in I(N, k)}$, so that we may consistently confuse $\Lambda^{k} \mathbf{R}_{\tilde{x}}^{N}$ with $\mathbf{R}_{y}^{d}$, where

$$
d:=\left(\begin{array}{c}
N \\
k
\end{array}\right) .
$$

The projection operators from the product space $\mathbf{R}_{x}^{N} \times \Lambda^{k} \mathbf{R}_{\tilde{x}}^{N}$ onto the single factor spaces $\mathbf{R}_{x}^{N}$ and $\Lambda^{k} \mathbf{R}_{\tilde{x}}^{N}$ will be denoted $p$ and $q$, respectively.

The linear subspace associated to a non zero simple multivector $\tau$ will be called "the enveloping subspace of $\tau$ " (according to [16]).

Both the standard inner product and the dual pairing for all the vector spaces we will deal with will be denoted by $\langle\cdot, \cdot\rangle$. For example, if $\eta, \tau \in$ $\Lambda^{n}\left(\mathbf{R}_{x}^{N}\right)$

$$
\langle\eta, \tau\rangle:=\sum_{\alpha}\left\langle\eta, e_{\alpha}\right\rangle\left\langle\tau, e_{\alpha}\right\rangle=\sum_{\alpha} \eta^{\alpha} \tau^{\alpha}
$$


The only notion of "length" we will need is the natural one induced by $\langle\cdot, \cdot\rangle$, namely:

$$
|\cdot|:=\sqrt{\langle\cdot, \cdot\rangle}
$$

For $h=1, \ldots, n$, the stratum $h$ of a vector

$$
\zeta \in \Lambda^{n}\left(\mathbf{R}_{x}^{N} \times \mathbf{R}_{y}^{d}\right)
$$

is defined as follows:

$$
\zeta_{(h)}:=\sum_{\substack{\gamma \in I(N, n-h) \\ \delta \in I(N, h)}}\left\langle\zeta, e_{\gamma} \wedge \varepsilon_{\delta}\right\rangle e_{\gamma} \wedge \varepsilon_{\delta}
$$

Throughout the paper we will consider at most the strata 0 and 1 of the multi-vectors we will deal with. For example, we will need the following measures related to a current $T=\llbracket G, \eta, \theta \rrbracket \in \mathcal{R}_{n}\left(\mathbf{R}_{x}^{N} \times \Lambda^{k} \mathbf{R}_{\tilde{x}}^{N}\right)$ :

$$
\left|T_{(h)}\right|:=\left|\eta_{(h)}\right||T|=\left|\eta_{(h)}\right| \theta \mathcal{H}^{n}\llcorner G
$$

for $h=0,1$. Obviously, such a definition makes sense for all $h=0,1, \ldots, n$.

Finally we will adopt the standard inclusion and equality symbols to denote the corresponding notions "in measure". For example, if $A$ and $B$ are $\mathcal{H}^{h}$-measurable sets, then " $A \subset B$ " will mean $\mathcal{H}^{h}(A \backslash B)=0$.

\section{Generalized Gauss graphs: Some well-known fact.}

Let us begin this section by recalling the definition of generalized Gauss graph.

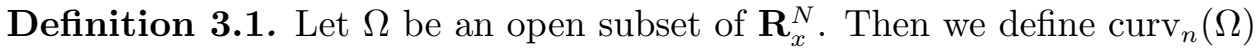
as the set of currents $T \in \mathcal{D}_{n}\left(\Omega \times \Lambda^{k} \mathbf{R}_{\tilde{x}}^{N}\right)$ such that:

(i) $T$ and $\partial T$ are rectifiable and supported in $\Omega \times S_{1}\left(\Lambda^{k} \mathbf{R}_{\tilde{x}}^{N}\right)$;

(ii) $\left\langle T\left\llcorner\lambda, \varphi\llcorner\omega\rangle=0\right.\right.$ for all $\lambda \in \mathcal{D}^{n-1}\left(\Omega \times \Lambda^{k} \mathbf{R}_{\tilde{x}}^{N}\right)$ and $\omega \in \mathcal{D}^{k-1}(\Omega \times$ $\left.\Lambda^{k} \mathbf{R}_{\tilde{x}}^{N}\right)$, where $\varphi(x, y):=\sum_{\beta \in I(N, k)} y^{\beta} d x^{\beta}$

(iii) $\left\langle\partial T\left\llcorner\mu, \varphi\llcorner\omega\rangle=0\right.\right.$ for all $\mu \in \mathcal{D}^{n-2}\left(\Omega \times \Lambda^{k} \mathbf{R}_{\tilde{x}}^{N}\right)$ and $\omega \in \mathcal{D}^{k-1}(\Omega \times$ $\left.\Lambda^{k} \mathbf{R}_{\tilde{x}}^{N}\right)$

(iv) $\quad(-1)^{k n}\left\langle T\llcorner\star \varphi, g\rangle \geq 0\right.$ for all non negative $g \in C_{c}^{0}\left(\Omega \times \Lambda^{k} \mathbf{R}_{\tilde{x}}^{N}\right)$, where $\star$ is the Hodge operator in $\mathbf{R}_{x}^{N}$ with respect to the canonical basis, so that $\star \varphi(x, y)=\sum_{\beta \in I(N, k)} \sigma(\beta, \bar{\beta}) y^{\beta} d x^{\bar{\beta}}$.

A current belonging to $\operatorname{curv}_{n}(\Omega)$ will be called a "generalized Gauss graph".

The following remark explains why the foregoing definition extends the classical notion of Gauss graph.

Remark 3.1. Let $M$ be a $n$-dimensional surface, embedded in $\mathbf{R}_{x}^{N}$, smooth, oriented and with smooth boundary $\partial M \subset M$. We say that a $n$-dimensional 
surface $G \subset \mathbf{R}_{x}^{N} \times S_{1}\left(\Lambda^{k} \mathbf{R}_{\tilde{x}}^{N}\right)$ is a Gauss graph of $M$ when it is the graph of $\star \tau$, where $\tau$ is a smooth orientation of $M$ i.e. a smooth field of unit simple $n$-vectors tangent to $M$. Obviously, there exist only two Gauss graphs of $M$, namely, given a smooth orientation $\tau$, the graphs of $\star \tau$ and $-\star \tau$.

Note that, given a Gauss graph $G$ of $M$, one can immediately recover the smooth orientation $\tau$ such that $G$ is the graph of $\star \tau$. In fact

$$
\tau(x)=(-1)^{k n} \star y
$$

is true on $G$ since $y=\star \tau(x)$ has to hold at all $(x, y) \in G$. Now, denoting by $\nu$ a smooth extension to $\mathbf{R}_{x}^{N}$ of the map that associates $x \in M$ with the unique $y$ such that $(x, y) \in G$ (so that $\nu_{\mid M}=\star \tau$ ), it is natural to consider the one multiplicity rectifiable current $T$ carried by $G$ and oriented by

$$
\eta:=\frac{\Lambda^{n}(I \oplus d \tilde{\nu}) \tau}{\left|\Lambda^{n}(I \oplus d \tilde{\nu}) \tau\right|} \circ p
$$

i.e.,

$$
T=\llbracket G, \eta, 1 \rrbracket .
$$

It is easy to check that $T \in \operatorname{curv}_{n}\left(\mathbf{R}_{x}^{N}\right)$. Indeed, the orthogonality between the enveloping spaces of $y$ and $\eta(x, y)$, at all $(x, y) \in G$, is just expressible in terms of $T$ by means of conditions (ii) and (iii) in Definition 3.1 (see also Proposition 3.1(1) below), while (iv) is clearly fulfilled just thanks to the choice, suitably made, of the orientation $\eta$. Furthermore, any current of the form $\llbracket G, \sigma \eta, 1 \rrbracket$, where $\sigma: G \rightarrow\{ \pm 1\}$ is $\mathcal{H}^{n}$ measurable, continues to verify (ii) and (iii) but only $T$, among them, satisfies (iv).

The following couple of propositions recalls all we shall need in this paper about generalized Gauss graphs. For a more exhaustive presentation of the subject see [1], [5], [8] and [9].

Proposition 3.1. Let $T=\llbracket G, \eta, \theta \rrbracket \in \operatorname{curv}_{n}(\Omega)$. Then:

(1) the condition (ii) in Definition 3.1 is fulfilled if and only if the enveloping subspaces of $\eta(x, y)$ and $\sum_{\beta}\left\langle y, \varepsilon_{\beta}\right\rangle e_{\beta}$ are mutually orthogonal in $\mathbf{R}_{x}^{N} \times \Lambda^{k} \mathbf{R}_{\tilde{x}}^{N}$ at $\mathcal{H}^{n}$-a.e. $(x, y) \in G$;

(2) for $\mathcal{H}^{n}$-a.e. $(x, y) \in G$, one has $(-1)^{k n} \eta_{(0)}(x, y)=\left|\eta_{(0)}(x, y)\right| \star y$;

(3) there exists a $\mathcal{H}^{n}$-measurable map $v: p G \rightarrow S_{1}\left(\Lambda^{k} \mathbf{R}_{x}^{N}\right)$ such that, for $\mathcal{H}^{n}$ a.e. $x \in p G, v(x)$ is simple, its enveloping subspace is orthogonal to the tangent subspace of $p G$ at $x$ and $p_{\mid G}^{-1}(x) \subset\{(x, v(x)),(x,-v(x))\}$.

Proposition 3.2. Assume $T, T_{j}(j=1,2, \ldots)$ belong to $\operatorname{curv}_{n}(\Omega)$ and be such that $T_{j} \rightarrow T$. Then $\left|T_{j(0)}\right| \rightarrow\left|T_{(0)}\right|$. 


\section{The factors of a generalized Gauss graph are generalized Gauss graphs themselves.}

Definition 4.1. We say that a countable family $\left\{T_{j}\right\} \subset \mathcal{D}_{n}\left(\mathbf{R}^{M}\right)$ is a proper decomposition of $T \in \mathcal{D}_{n}\left(\mathbf{R}^{M}\right)$ if

$$
T=\sum_{j} T_{j} \quad \text { and } \quad \mathbf{N}(T)=\sum_{j} \mathbf{N}\left(T_{j}\right) .
$$

The currents $T_{j}$ will be called factors of $T$.

We recall from $[11,4.2 .25]$ that every $T \in \mathbf{I}_{n}\left(\mathbf{R}^{M}\right)$ has a proper decomposition whose factors are indecomposable currents in $\mathbf{I}_{n}\left(\mathbf{R}^{M}\right)$.

This section is devoted to prove that every factor of a null boundary generalized Gauss graph has to be a null boundary generalized Gauss graph itself.

Proposition 4.1. Let $T, T_{j}(j=1,2, \ldots)$ be in $\mathcal{D}_{n}\left(\mathbf{R}^{M}\right)$ such that

$$
\partial T=0 \quad \text { and } \quad T=\sum_{j} T_{j} .
$$

Then

$$
\mathbf{N}(T)=\sum_{j} \mathbf{N}\left(T_{j}\right)
$$

if and only if

$$
\mathbf{M}(T)=\sum_{j} \mathbf{M}\left(T_{j}\right) \quad \text { and } \quad \partial T_{j}=0 \text { for all } j .
$$

Proof. If $\mathbf{N}(T)=\sum_{j} \mathbf{N}\left(T_{j}\right)$ holds, then one has also

$$
\sum_{j} \mathbf{N}\left(T_{j}\right)=\mathbf{N}(T)=\mathbf{M}(T) \leq \sum_{j} \mathbf{M}\left(T_{j}\right)
$$

i.e., $\sum_{j} \mathbf{M}\left(\partial T_{j}\right) \leq 0$, whence we conclude the proof of "only if ". The vice versa is trivial.

Proposition 4.2. Let $T=\llbracket G, \eta, \theta \rrbracket, T_{j}=\llbracket G_{j}, \eta_{j}, \theta_{j} \rrbracket(j=1,2, \ldots)$ be in $\mathcal{R}_{n}\left(\mathbf{R}^{M}\right)$ such that

$$
T=\sum_{j} T_{j} \quad \text { and } \quad \mathbf{M}(T)=\sum_{j} \mathbf{M}\left(T_{j}\right) .
$$

Then one has:

(1) $G=\cup_{j} G_{j}$ 
(2) $\eta_{\mid G_{j}}=\eta_{j}(j=1,2, \ldots)$ and $\theta=\sum_{j} \tilde{\theta}_{j}$, where

$$
\tilde{\theta}_{j}:= \begin{cases}\theta_{j} & \text { on } G_{j} \\ 0 & \text { on } G \backslash G_{j} .\end{cases}
$$

Proof. Let us consider the family of $\mathcal{H}^{n}$-rectifiable sets defined as follows:

$$
G_{(\beta)}:=\left(G_{\beta_{1}} \cap \ldots \cap G_{\beta_{l}}\right) \backslash \cup_{j \notin \beta} G_{j}
$$

for all $\beta \in I(\infty)$. They constitute a "natural partition" of $G$ and, as one can check by a simple calculation, they satisfy the following conditions:

$$
\begin{gathered}
\cup_{\beta} G_{(\beta)}=\cup_{j} G_{j} \quad \text { and } \quad G_{(\beta)} \cap G_{\left(\beta^{\prime}\right)}=\emptyset \text { whenever } \beta \neq \beta^{\prime} \\
\sum_{j} \mathbf{M}\left(T_{j}\right)=\sum_{l=1}^{\infty} \sum_{\beta \in I(\infty, l)} \int_{G_{(\beta)}} \sum_{i=1}^{l} \theta_{\beta_{i}} d \mathcal{H}^{n} \\
\sum_{j} T_{j}(\omega)=\sum_{l=1}^{\infty} \sum_{\beta \in I(\infty, l)} \int_{G_{(\beta)}}\left\langle\eta_{(\beta)}, \omega\right\rangle \theta_{(\beta)} d \mathcal{H}^{n}
\end{gathered}
$$

for all $\omega \in \mathcal{D}^{n}\left(\mathbf{R}^{M}\right)$, where:

$$
\left\{\begin{array}{l}
\eta_{(\beta)}:=\eta_{\beta_{1}} \\
\theta_{(\beta)}:=\theta_{\beta_{1}}+\left\langle\eta_{\beta_{2}}, \eta_{\beta_{1}}\right\rangle \theta_{\beta_{2}}+\ldots+\left\langle\eta_{\beta_{l}}, \eta_{\beta_{1}}\right\rangle \theta_{\beta_{l}} .
\end{array}\right.
$$

As a consequence, we have:

$$
\begin{aligned}
& \int_{G}(1-\langle\eta, \omega\rangle) \theta d \mathcal{H}^{n}=\mathbf{M}(T)-T(\omega)=\sum_{j} \mathbf{M}\left(T_{j}\right)-\sum_{j} T_{j}(\omega) \\
& =\sum_{l=1}^{\infty} \sum_{\beta \in I(\infty, l)} \int_{G_{(\beta)}} \sum_{i=1}^{l} \theta_{\beta_{i}}\left(1-\left\langle\eta_{\beta_{1}}, \omega\right\rangle\left\langle\eta_{\beta_{i}}, \eta_{\beta_{1}}\right\rangle\right) d \mathcal{H}^{n} \\
& =\sum_{l=1}^{\infty} \sum_{\beta \in I(\infty, l)} \int_{G_{(\beta)}} \sum_{i=1}^{l} \theta_{\beta_{i}}\left(1-\left\langle\eta_{\beta_{i}}, \omega\right\rangle\right) d \mathcal{H}^{n}
\end{aligned}
$$

for all $\omega \in \mathcal{D}^{n}\left(\mathbf{R}^{M}\right)$. Hence, by localizing, it follows that

$$
G_{(\beta)} \subset G
$$

for all $\beta$.

We remark that this conclusion cannot be drawn directly from (4.4) since $\theta_{(\beta)}$ could be zero somewhere. 
By (4.5) and (4.2), one obtains:

$$
\cup_{j} G_{j}=\cup_{\beta} G_{(\beta)} \subset G
$$

and then, as the opposite inclusion is obvious, the proof of (1) is concluded.

To prove (2), let us define

$$
\sigma_{j}: G \rightarrow\{0, \pm 1\}
$$

as follows:

$$
\sigma_{j}:= \begin{cases}\left\langle\eta, \eta_{j}\right\rangle \in\{ \pm 1\} & \text { on } G_{j} \\ 0 & \text { on } G \backslash G_{j} .\end{cases}
$$

As $G_{j} \subset G$ and (4.1) holds, one has:

$$
\int_{G}\langle\eta, \omega\rangle \theta d \mathcal{H}^{n}=\sum_{j} \int_{G_{j}}\left\langle\eta_{j}, \omega\right\rangle \theta_{j} d \mathcal{H}^{n}=\int_{G}\langle\eta, \omega\rangle \sum_{j} \sigma_{j} \tilde{\theta}_{j} d \mathcal{H}^{n}
$$

for all $\omega \in \mathcal{D}^{n}\left(\mathbf{R}^{M}\right)$. By the arbitrariness of $\omega$, it follows that

$$
\theta=\sum_{j} \sigma_{j} \tilde{\theta}_{j}
$$

$\mathcal{H}^{n}\llcorner G$ - a.e. On the other hand, by (4.1) again, one has also

$$
\int_{G} \theta d \mathcal{H}^{n}=\sum_{j} \int_{G_{j}} \theta_{j} d \mathcal{H}^{n}
$$

and thus

$$
\sum_{j} \int_{G_{j}} \sigma_{j} \theta_{j} d \mathcal{H}^{n}=\int_{G} \sum_{j} \sigma_{j} \tilde{\theta}_{j} d \mathcal{H}^{n}=\sum_{j} \int_{G_{j}} \theta_{j} d \mathcal{H}^{n}
$$

i.e.

$$
\sum_{j} \int_{G_{j}}\left(1-\sigma_{j}\right) \theta_{j} d \mathcal{H}^{n}=0 .
$$

Now (2) becomes evident by recalling (4.6) again.

Proposition 4.3. Let $T \in \operatorname{curv}_{n}\left(\mathbf{R}_{x}^{N}\right), \partial T=0$ and let $\left\{T_{j}\right\} \subset \mathcal{R}_{n}\left(\mathbf{R}_{x}^{N} \times\right.$ $\left.\Lambda^{k} \mathbf{R}_{\tilde{x}}^{N}\right)$ be a proper decomposition of $T$. Then

$$
T_{j} \in \operatorname{curv}_{n}\left(\mathbf{R}_{x}^{N}\right) \quad \text { and } \quad \partial T_{j}=0
$$

for all $j$. 
Proof. Let us note that $\partial T_{j}=0$ immediately follows from Proposition 4.1 which implies also that

$$
\mathbf{M}(T)=\sum_{j} \mathbf{M}\left(T_{j}\right)
$$

Then we can apply Proposition 4.2 to obtain

$$
G_{j} \subset G \quad \text { and } \quad \eta_{\mid G_{j}}=\eta_{j}
$$

for all $j$. Hence, by Proposition 3.1, it follows that the enveloping subspaces of $\eta(x, y)$ and $y$ are mutually orthogonal at $\mathcal{H}^{n}\left\llcorner G_{j}\right.$ almost every $(x, y)$, for all $j$. In other words: The hypothesis (ii) of Definition 3.1 is fulfilled by all $T_{j}$.

To conclude, we have only to check that also (iv) of Definition 3.1 is satisfied. But this easily follows by using again (4.7) together with Proposition 3.1, as the calculation below shows (let $g \in C_{c}^{0}\left(\mathbf{R}_{x}^{N} \times \Lambda^{k} \mathbf{R}_{\tilde{x}}^{N}\right)$ ):

$$
\begin{aligned}
(-1)^{k n}\left\langle T_{j}\llcorner\star \varphi, g\rangle\right. & =(-1)^{k n} \int_{G_{j}}\left\langle\eta_{j}, \star \varphi\right\rangle g \theta_{j} d \mathcal{H}^{n} \\
& =(-1)^{k n} \int_{G_{j}}\langle\eta, \star \varphi\rangle g \theta_{j} d \mathcal{H}^{n}=\int_{G_{j}}\left|\eta_{(0)}\right| g \theta_{j} d \mathcal{H}^{n} .
\end{aligned}
$$

Remark 4.1. Let us assume the hypotheses of Proposition 4.2 be verified. Then the following two simple consequences of the proposition can be given.

First.

$$
\left|T_{j_{(1)}}\right| \ll\left|T_{j_{(0)}}\right|
$$

for all $j$, whenever $\left|T_{(1)}\right| \ll\left|T_{(0)}\right|$. This fact, along with Proposition 4.3, is interesting with regard to the links between generalized Gauss graphs and curvature varifolds. More precisely, if $\left|T_{(1)}\right| \ll\left|T_{(0)}\right|$ holds then $T$ and all its factors $T_{j}$ induce curvature varifolds (see [9] for details).

Second. Let

$$
f: \mathbf{R}_{x}^{N} \times \Lambda^{k} \mathbf{R}_{\tilde{x}}^{N} \times \Lambda^{n}\left(\mathbf{R}_{x}^{N} \times \Lambda^{k} \mathbf{R}_{\tilde{x}}^{N}\right) \rightarrow[-\infty,+\infty]
$$

be a Borel measurable function such that

$$
\int|f(x, y, \eta(x, y))| d \mathcal{H}^{n}\llcorner G<+\infty .
$$

Then we can define the real measure

$$
\mu(\cdot):=\int f(x, y, \eta(x, y)) d \mathcal{H}^{n}\llcorner G
$$


and hence also the positive and negative variations of $\mu$ can be defined: Let us denote them by $\mu^{+}$and $\mu^{-}$(see, for example, [19] as a general reference).

If we assume that

$$
\theta \in L^{1}\left(\mu^{+}\right) \quad \text { or } \quad \theta \in L^{1}\left(\mu^{-}\right)
$$

then, by Proposition 4.2, we get

$$
\int_{G} f(x, y, \eta(x, y)) \theta(x, y) d \mathcal{H}^{n}=\sum_{j} \int_{G_{j}} f\left(x, y, \eta_{j}(x, y)\right) \theta_{j}(x, y) d \mathcal{H}^{n}
$$

as the following computation shows:

$$
\begin{aligned}
\int_{G} f(x, y, \eta(x, y)) \theta(x, y) d \mathcal{H}^{n} & =\int \theta d \mu=\int \theta d \mu^{+}-\int \theta d \mu^{-} \\
& =\sum_{j} \int \tilde{\theta}_{j} d \mu^{+}-\sum_{j} \int \tilde{\theta}_{j} d \mu^{-}=\sum_{j} \int \tilde{\theta}_{j} d \mu \\
& =\sum_{j} \int_{G_{j}} f\left(x, y, \eta_{j}(x, y)\right) \theta_{j}(x, y) d \mathcal{H}^{n} .
\end{aligned}
$$

When $f \geq 0$, Proposition 4.2 and monotone convergence theorem imply that (4.8) holds without any further condition on $\theta$, even if

$$
\int f(x, y, \eta(x, y)) d \mathcal{H}^{n}\llcorner G=+\infty .
$$

In particular, taking $f \equiv 1$, one recovers the mass condition in (4.1).

\section{The problem.}

Given a $n$-dimensional rectifiable set $M \subset \mathbf{R}^{N}$, a non negative constant $c$ and a current $B \in \mathcal{R}_{n-1}\left(\mathbf{R}_{x}^{N} \times \Lambda^{k} \mathbf{R}_{\tilde{x}}^{N}\right)$, one can define the class of currents

$$
\mathcal{I}_{B}^{M, c}:=\left\{T \in \operatorname{curv}_{n}\left(\mathbf{R}^{N}\right) \mid \mathbf{M}(\partial T-B) \leq c \text { and } p(\operatorname{spt} T) \supset M\right\} .
$$

Then we can consider the problem of minimizing a sufficiently nice functional $\mathcal{F}$ (for example the mass) among the currents belonging to $\mathcal{I}_{B}^{M, c}$.

Before going into details, let us give the following definition.

Definition 5.1. Let us be given a functional $\mathcal{F}$ defined on a subset $\Sigma$ of $\mathcal{D}^{n}\left(\mathbf{R}_{x}^{N} \times \Lambda^{k} \mathbf{R}_{\tilde{x}}^{N}\right)$. Then we say that:

(1) $\mathcal{F}$ is lower semi-continuous if

$$
\mathcal{F}(T) \leq \liminf _{j} \mathcal{F}\left(T_{j}\right)
$$

whenever $T_{j} \rightarrow T\left(T, T_{j} \in \Sigma\right)$. 
(2) $\mathcal{F}$ is coercive if in every set $S \subset \Sigma$ such that $\mathcal{F}(S)$ is bounded there exists a sequence that converges weakly to an element of $S$.

We shall show that, if $\mathcal{F}$ is lower semi-continuous and coercive, then the problem above has a solution. First of all we need the stability of the condition

$$
p(\operatorname{spt} T) \supset M
$$

with respect to the weak convergence in $\operatorname{curv}_{n}\left(\mathbf{R}^{N}\right)$.

Proposition 5.1. Let $T, T_{j} \in \operatorname{curv}_{n}\left(\mathbf{R}^{N}\right)$ and let $M$ be a n-dimensional rectifiable subset of $\mathbf{R}^{N}$. If

$$
T_{j} \rightarrow T \quad \text { and } \quad p\left(\operatorname{spt} T_{j}\right) \supset M
$$

then one has also $p(\operatorname{spt} T) \supset M$.

Proof. Let $T=\llbracket G, \eta, \theta \rrbracket, T_{j}=\llbracket G_{j}, \eta_{j}, \theta_{j} \rrbracket$ and $g \in C_{c}^{0}\left(\mathbf{R}^{N}\right)$. Then, by the area formula and Proposition 3.2, we get:

$$
\begin{aligned}
& \int_{p G} g(x) \sum_{(x, y) \in p^{-1}(x) \cap G} \theta(x, y) d \mathcal{H}^{n}(x, y)=\int_{G} g(x)\left|\eta_{(0)}(x, y)\right| \theta(x, y) d \mathcal{H}^{n}(x, y) \\
& =\lim _{j} \int_{G_{j}} g(x)\left|\eta_{j(0)}(x, y)\right| \theta_{j}(x, y) d \mathcal{H}^{n}(x, y) \\
& =\lim _{j} \int_{p G_{j}} g(x) \sum_{(x, y) \in p^{-1}(x) \cap G_{j}} \theta_{j}(x, y) d \mathcal{H}^{n}(x, y) .
\end{aligned}
$$

In particular, one has

$$
\int_{p G} g(x) \sum_{(x, y) \in p^{-1}(x) \cap G} \theta(x, y) d \mathcal{H}^{n}(x, y) \geq \int_{M} g d \mathcal{H}^{n}
$$

for all non negative $g \in C_{c}^{0}\left(\mathbf{R}^{N}\right)$. Now the thesis follows by an usual localization argument.

Remark 5.1. In Proposition 5.1 the hypothesis $T_{j} \in \operatorname{curv}_{n}\left(\mathbf{R}^{N}\right)$ is crucial in the sense that the thesis can be false if one assumes only that $\operatorname{spt} T_{j} \subset$ $\mathbf{R}_{x}^{N} \times S_{1}\left(\Lambda^{k} \mathbf{R}_{\tilde{x}}^{N}\right)$. Here is an example.

Example $(n=k=1)$. Let $\sigma$ be a straight segment in $\mathbf{R}^{2}$ and let $\left\{u_{j}\right\} \subset$ $S_{1}\left(\mathbf{R}^{2}\right)$ be a sequence converging to $u$. If $T_{j}$ is the one dimensional, multiplicity one, rectifiable current carried by the couple of segments $\sigma \times\{u\}$ and $\sigma \times\left\{u_{j}\right\}$ endowed with opposite orientations, then one has spt $T_{j} \subset$ $\mathbf{R}^{2} \times S_{1}\left(\mathbf{R}^{2}\right), \mathrm{p}\left(\operatorname{spt} T_{j}\right)=\sigma$ and $T_{j} \rightarrow T:=0$. 
Now, by the direct method, we easily obtain the existence result.

Proposition 5.2. Let $\mathcal{I}_{B}^{M, c}$ be not empty and assume $\mathcal{F}: \mathcal{I}_{B}^{M, c} \rightarrow[0,+\infty]$ be lower semi-continuous and coercive. Then there exists a minimizer of $\mathcal{F}$.

Proof. If

$$
m:=\inf _{\mathcal{I}_{B}^{M, c}} \mathcal{F}=+\infty
$$

then there is nothing to prove. Otherwise, let $\left\{T_{j}\right\}$ be a minimizing sequence. As $\mathcal{F}$ is coercive, we can extract a subsequence which converges weakly to $T \in \operatorname{curv}_{n}\left(\mathbf{R}^{N}\right)$. By Proposition 5.1 one has $T \in \mathcal{I}_{B}^{M, c}$ and finally, as $\mathcal{F}$ is lower semi-continuous, $\mathcal{F}(T)=m$.

Examples. Both the following situations correspond to problems solvable by means of Proposition 5.2:

Situation 1: $\mathcal{F}=\mathbf{M}$ and $c=0$ (here we are dealing with a prescribed boundary problem).

Situation 2: $\mathcal{F}=\mathbf{M}$ and $B=0$.

Remark 5.2. Natural examples of functionals to consider are the integral ones

$$
\operatorname{curv}_{n}\left(\mathbf{R}^{N}\right) \ni T=\llbracket G, \eta, \theta \rrbracket \rightarrow \mathcal{E}(T):=\int_{G} f(x, y, \eta(x, y)) \theta(x, y) d \mathcal{H}^{n}(x, y)
$$

where

$$
f: \mathbf{R}_{x}^{N} \times \Lambda^{k} \mathbf{R}_{\tilde{x}}^{N} \times S_{1}\left(\Lambda^{n}\left(\mathbf{R}_{x}^{N} \times \Lambda^{k} \mathbf{R}_{\tilde{x}}^{N}\right)\right) \rightarrow[-\infty,+\infty]
$$

has to satisfy some conditions that allow $\mathcal{E}$ to be nice for our minimum problem. For example, if $f$ is Borel measurable and $f \geq \varepsilon>0$ then $\mathcal{E}$ is coercive.

In order that $\mathcal{E}$ be lower semi-continuous, one can assume, for example, the "adequateness" condition on $f$ (introduced in [5], see also [7]).

Finally we obtain the following result.

Proposition 5.3. Let $\mathcal{I}_{B}^{M, c}$ be not empty and assume $f$ be adequate and $f \geq \varepsilon>0$. Then the functional $\mathcal{E}_{\mid \mathcal{I}_{B}^{M, c}}$ has a minimizer. 


\section{A factor of a minimizer is a minimizer.}

Let $M$ be a $n$-dimensional rectifiable subset of $\mathbf{R}_{x}^{N}$ such that $\mathcal{I}_{0}^{M, 0}$ is non empty and consider a functional

$$
\mathcal{G}: \mathcal{I}_{0}^{\emptyset, 0}=\left\{T \in \operatorname{curv}_{n}\left(\mathbf{R}^{N}\right) \mid \partial T=0\right\} \rightarrow[-\infty,+\infty]
$$

such that the restriction

$$
\mathcal{F}:=\mathcal{G}_{\mid \mathcal{I}_{0}^{M, 0}}
$$

has a minimizer $T$. For example this is true when $\mathcal{F}$ is non negative, lower semi-continuous and coercive, by Proposition 5.2.

If $\left\{T_{j}\right\} \subset \mathcal{R}_{n}\left(\mathbf{R}_{x}^{N} \times \Lambda^{k} \mathbf{R}_{\tilde{x}}^{N}\right)$ is a proper decomposition of $T$, then Proposition 4.3 implies that

$$
T_{j} \in \operatorname{curv}_{n}\left(\mathbf{R}^{N}\right) \quad \text { and } \quad \partial T_{j}=0
$$

for all $j$, whereby one can wonder if $T_{j}$ minimizes the functional

$$
\mathcal{F}_{j}:=\mathcal{G}_{\mid I_{0}^{M_{j}, 0}}
$$

where $M_{j}:=M \cap p\left(\operatorname{spt} T_{j}\right)$.

The following proposition gives an answer.

Proposition 6.1. Let $\mathcal{G}$ be non negative and subadditive. Moreover, assume that

$$
\mathcal{G}(T)=\sum_{j} \mathcal{G}\left(T_{j}\right)
$$

Then $T_{j}$ minimizes $\mathcal{F}_{j}$, for all $j$.

Proof. It is enough to prove the thesis for $j=1$.

Furthermore, one can assume that the decomposition consists of just two factors since also

$$
\mathcal{G}(T)=\mathcal{G}\left(T_{1}\right)+\mathcal{G}\left(\sum_{j \geq 2} T_{j}\right)
$$

has to hold, as the following computation shows:

$$
\mathcal{G}(T)=\sum_{j} \mathcal{G}\left(T_{j}\right)=\mathcal{G}\left(T_{1}\right)+\sum_{j \geq 2} \mathcal{G}\left(T_{j}\right) \geq \mathcal{G}\left(T_{1}\right)+\mathcal{G}\left(\sum_{j \geq 2} T_{j}\right) \geq \mathcal{G}(T)
$$

Similarly, we have also

$$
\mathbf{N}(T)=\mathbf{N}\left(T_{1}\right)+\mathbf{N}\left(\sum_{j \geq 2} T_{j}\right)
$$


whereby

$$
\left\{T_{1}, \sum_{j \geq 2} T_{j}\right\}
$$

is a proper decomposition of $T$. So the thesis is reduced to the following one: If $\left\{T_{1}, T_{2}\right\}$ is a proper decomposition of $T$ such that $\mathcal{G}(T)=\mathcal{G}\left(T_{1}\right)+\mathcal{G}\left(T_{2}\right)$, then $T_{1}$ minimizes $\mathcal{F}_{1}$, i.e.,

$$
\mathcal{F}_{1}\left(T_{1}\right) \leq \mathcal{F}_{1}\left(T_{1}^{\prime}\right)
$$

for all $T_{1}^{\prime} \in \mathcal{I}_{0}^{M_{1}, 0}$.

In order to prove it, let us take $T_{1}^{\prime} \in \mathcal{I}_{0}^{M_{1}, 0}$ and note that

$$
\Sigma:=T+T_{1}^{\prime}-T_{1}=T_{2}+T_{1}^{\prime} \in \mathcal{I}_{0}^{M, 0} .
$$

In fact, one has

$$
\Sigma \in \operatorname{curv}_{n}\left(\mathbf{R}^{N}\right) \quad \text { and } \quad \partial \Sigma=0
$$

by (6.1), while

$$
p(\operatorname{spt} \Sigma) \supset M
$$

holds by Proposition 6.2 below. As $T$ is a minimizer of $\mathcal{F},(6.3)$ implies that

$$
\mathcal{F}(T) \leq \mathcal{F}(\Sigma)
$$

Hence

$$
\mathcal{G}\left(T_{1}\right)+\mathcal{G}\left(T_{2}\right) \leq \mathcal{G}\left(T_{2}\right)+\mathcal{G}\left(T_{1}^{\prime}\right)
$$

i.e.,

$$
\mathcal{G}\left(T_{1}\right) \leq \mathcal{G}\left(T_{1}^{\prime}\right)
$$

that is just (6.2).

Now let us state and prove the result used in the proof of Proposition 6.1.

Proposition 6.2. Let there be given a rectifiable $n$-dimensional set $M \subset$ $\mathbf{R}^{N}$ and $T, T_{1}, T_{2}, T_{1}^{\prime} \in \mathcal{R}_{n}\left(\mathbf{R}_{x}^{N} \times S_{1}\left(\Lambda^{k} \mathbf{R}_{\tilde{x}}^{N}\right)\right)$ be such that the following hypotheses hold:

(i) $\quad T=T_{1}+T_{2}$

(ii) $(-1)^{k n} T_{2}\left\llcorner\star \varphi=\left|T_{2(0)}\right|\right.$ and $(-1)^{k n} T_{1}^{\prime}\left\llcorner\star \varphi=\left|T_{1(0)}^{\prime}\right|\right.$

(iii) $\quad p(\operatorname{spt} T) \supset M$ 
(iv) $p\left(\operatorname{spt} T_{1}^{\prime}\right) \supset M \cap p\left(\operatorname{spt} T_{1}\right)$.

Then one has

$$
p\left(\operatorname{spt}\left(T_{2}+T_{1}^{\prime}\right)\right) \supset M
$$

Proof. For the sake of shortness we shall adopt the following notation:

$$
\left\{\begin{array}{l}
P:=p(\operatorname{spt} T) \text { and } P_{1}^{\prime}:=p\left(\operatorname{spt} T_{1}^{\prime}\right) \\
P_{i}:=p\left(\operatorname{spt} T_{i}\right) \text { and } M_{i}:=M \cap P_{i}, \quad \text { for } i=1,2 .
\end{array}\right.
$$

Let $T_{2}=\llbracket G_{2}, \eta_{2}, \theta_{2} \rrbracket$ and $T_{1}^{\prime}=\llbracket G_{1}^{\prime}, \eta_{1}^{\prime}, \theta_{1}^{\prime} \rrbracket$. Then (ii) and the area formula imply that, for all non negative $g \in C_{c}^{0}\left(\mathbf{R}_{x}^{N}\right)$, one has:

$$
\begin{aligned}
& (-1)^{k n}\left(T_{2}+T_{1}^{\prime}\right)(g \star \varphi)=\left|T_{2(0)}\right|(g)+\left|T_{1(0)}^{\prime}\right|(g) \\
& =\int_{G_{2}} g\left|\eta_{2(0)}\right| \theta_{2} d \mathcal{H}^{n}+\int_{G_{1}^{\prime}} g\left|\eta_{1(0)}^{\prime}\right| \theta_{1}^{\prime} d \mathcal{H}^{n} \\
& =\int_{P_{2}} g(x) \sum_{(x, y) \in p^{-1}(x) \cap G_{2}} \theta_{2}(x, y) d \mathcal{H}^{n}(x, y)+ \\
& \quad+\int_{P_{1}^{\prime}} g(x) \sum_{(x, y) \in p^{-1}(x) \cap G_{1}^{\prime}} \theta_{1}^{\prime}(x, y) d \mathcal{H}^{n}(x, y) \geq \int_{P_{2} \cup P_{1}^{\prime}} g d \mathcal{H}^{n} .
\end{aligned}
$$

Hence the conclusion follows by localizing, once proved that

$$
P_{2} \cup P_{1}^{\prime} \supset M
$$

In order to verify such inclusion, we note that $M \subset P \subset P_{1} \cup P_{2}$ has to hold, by (i) and (iii). Thus, by (iv), we get:

$$
M=M \cap\left(P_{1} \cup P_{2}\right)=M_{1} \cup M_{2} \subset M_{1} \cup P_{2} \subset P_{1}^{\prime} \cup P_{2}
$$

i.e., just what we need.

The following proposition is an easy consequence of Proposition 5.3, Proposition 6.1 and Remark 4.1.

Proposition 6.3. Let $\mathcal{E}$ be the integral functional defined by means of an adequate function $f \geq \varepsilon>0$, as in Proposition 5.3. If $\mathcal{I}_{0}^{M, 0} \neq \emptyset$ then $\mathcal{E}_{\mid \mathcal{I}_{0}^{M, 0}}$ has a minimizer $T$. Moreover, every factor $T_{j}$ of a rectifiable proper decomposition of $T$ minimizes $\mathcal{E}_{\mid \mathcal{I}_{0}^{M_{j}, 0}}$, where $M_{j}:=M \cap p\left(\operatorname{spt} T_{j}\right)$.

Proof. Let us set

$$
\mathcal{G}:=\mathcal{E}_{\mid \mathcal{I}_{0}^{\emptyset, 0}} .
$$


By Proposition 5.3 we get a minimizer $T$ of the functional

$$
\mathcal{G}_{\mid \mathcal{I}_{0}^{M, 0}}=\mathcal{E}_{\mid \mathcal{I}_{0}^{M, 0}}
$$

Let $\left\{T_{j}\right\}$ be a rectifiable proper decomposition of $T$. We have

$$
\mathcal{G}(T)=\sum_{j} \mathcal{G}\left(T_{j}\right)
$$

by Remark 4.1. Moreover, as $f$ is non negative, one has also that $\mathcal{G}$ is non negative and subadditive. Then Proposition 6.1 implies that $T_{j}$ minimizes the functional

$$
\mathcal{G}_{\mid \mathcal{I}_{0}^{M_{j}, 0}}=\mathcal{E}_{\mid \mathcal{I}_{0}^{M_{j}, 0}}
$$

where $M_{j}:=M \cap p\left(\operatorname{spt} T_{j}\right)$.

\section{The case of the curves in the plane.}

This section is devoted to investigate the properties of a solution $T$ of our problem when $M$ is a closed and piecewise smooth curve in $\mathbf{R}^{2}$ and

$$
B=0, \quad c=0 \quad \text { and } \quad \mathcal{F}=\mathbf{M} .
$$

Note that $M$ has finite length, since $\mathcal{H}^{1}(M) \leq \mathbf{M}(T)$.

Let $S \subset M$ be the set of the singular points of $M$, namely the ones at which the tangent line to $M$ doesn't exist. If $M$ is simple then, by recalling Jordan's theorem (see for example [6]), one can define the outward normal map to $M$ :

$$
\nu: M \backslash S \rightarrow S_{1}\left(\mathbf{R}^{2}\right) .
$$

Let the graph of $\nu$ be denoted by $G_{\nu}$ and consider the rectifiable set $\tilde{G}_{\nu} \subset$ $M \times S_{1}\left(\mathbf{R}^{2}\right)$ obtained by filling the holes of $G_{\nu}$, over every $P \in S$, by means of an arc of $\{P\} \times S^{1}\left(\mathbf{R}^{2}\right)$ in the cheapest way (in the sense of the mass). As $G_{\nu}$ must have finite measure, $\tilde{G}_{\nu}$ has finite measure provided that the total length of the added arcs is finite. Analogously, by considering the inward normal in place of the outward one, we get $\tilde{G}_{-\nu}$.

The generalized Gauss graphs carried by $\tilde{G}_{\nu}$ and $\tilde{G}_{-\nu}$ will be called "natural Gauss graphs of $M "$.

Remark 7.1. One could hurriedly conclude that the natural Gauss graphs always have to be minimizers. The following example shows that this guess is false. 
Example (the double-comb). Let $C_{h}$ be the "double-comb" set pictured below respectively for $h=2$ and $h=3$
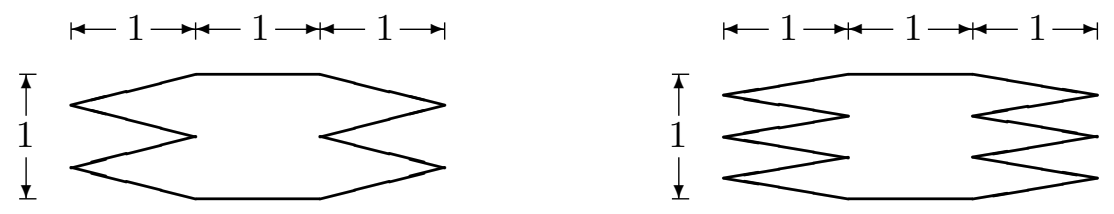

and let $C_{h}^{(j)}, j=1, \ldots, h$, denote the "double-teeth" that form $C_{h}$; here there is an explanatory figure, in the case $h=3$.

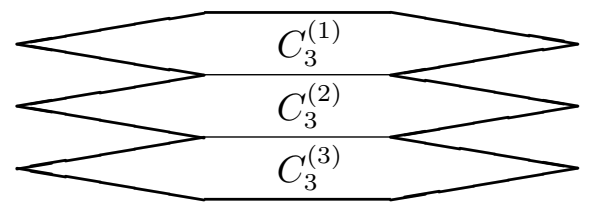

Then, let $\nu_{h}$ and $\nu_{h}^{(j)}$ be the outward normal map to $\partial C_{h}$ and $\partial C_{h}^{(j)}$ respectively and consider the multiplicity one generalized Gauss graphs $T_{h}$, $T_{h}^{(j)}$ carried by $\tilde{G}_{\nu_{h}}, \tilde{G}_{\nu_{h}^{(j)}}$ respectively (let us recall that they are uniquely determined, by the second statement in Proposition 3.1).

Furthermore let

$$
T_{h}^{\prime}:=\sum_{j=1}^{h} T_{h}^{(j)}
$$

It is obvious that

$$
T_{h}, T_{h}^{\prime} \in \mathcal{I}_{0}^{M_{h}, 0}
$$

where $M_{h}:=\partial C_{h}$. Moreover

$$
\mathbf{M}\left(T_{h}\right)>\mathbf{M}\left(T_{h}^{\prime}\right)
$$

for $h$ big enough. In fact:

$$
\mathbf{M}\left(T_{h}^{\prime}\right)=h \mathcal{H}^{1}\left(\tilde{G}_{\nu_{h}^{(1)}}\right)=h\left(2 \pi+2+4 \sqrt{1+\frac{1}{4 h^{2}}}\right)
$$

and

$$
\mathbf{M}\left(T_{h}\right)=\mathcal{H}^{1}\left(\tilde{G}_{\nu_{h}}\right)=2+4 h \sqrt{1+\frac{1}{4 h^{2}}}+2 \alpha_{h}+2\left(\pi-\alpha_{h}\right)(2 h-1)
$$

where $\alpha_{h}$ is the tooth angle.

We obtain

$$
\mathbf{M}\left(T_{h}\right)-\mathbf{M}\left(T_{h}^{\prime}\right)=2(h-1)\left(\pi-2 \alpha_{h}-1\right)
$$


whence the conclusion follows. By a simple calculation one can check that the same idea doesn't work if one takes the "more convex" simple-comb.

We shall prove that the convexity condition on $M$ is enough for the minimality of the natural Gauss graphs, just as one expects (probably a suitable weaker condition allowing "slightly nonconvex" $M$ 's could be sufficient).

Let us introduce some notation which will be useful in the rest of the section.

Let $S \subset \mathbf{R}_{x}^{2} \times \mathbf{R}_{y}^{2}$ be a $\mathcal{H}^{1}$-rectifiable set and define

$$
R_{S}^{+}:=G_{\nu} \cap S, \quad R_{S}^{-}:=Q\left(G_{\nu} \backslash R_{S}^{+}\right) \quad \text { and } \quad R_{S}:=R_{S}^{+} \cup R_{S}^{-}
$$

where $Q(x, y):=(x,-y)$. We set also

$$
M_{S}^{+}:=p R_{S}^{+} \quad \text { and } \quad M_{S}^{-}:=p R_{S}^{-} .
$$

Now we are ready to state the first proposition.

Proposition 7.1. Let $S \subset \mathbf{R}_{x}^{2} \times \mathbf{R}_{y}^{2}$ be a $\mathcal{H}^{1}$-rectifiable set. Then

(1) $\mathcal{H}^{1}\left(R_{S}\right)=\mathcal{H}^{1}\left(G_{\nu}\right)$.

Moreover, if $\Sigma \in \operatorname{curv}_{1}\left(\mathbf{R}^{2}\right)$ is nonzero and null-boundary then one has

(2) $\quad R_{\text {spt } \Sigma} \subset$ spt $\Sigma$, provided $p(\operatorname{spt} \Sigma) \supset M$;

(3) if $M$ is convex, then $\mathcal{H}^{1}\left(\operatorname{spt} \Sigma \backslash R_{S}\right) \geq \mathcal{H}^{1}\left(\tilde{G}_{\nu} \backslash G_{\nu}\right)$.

Proof. To prove the first statement, note that, as $R_{S}^{+}$and $R_{S}^{-}$are disjointed, as well as $R_{S}^{+}$and $Q R_{S}^{-}$which form a partition of $G_{\nu}$, one has

$$
\mathcal{H}^{1}\left(R_{S}\right)=\mathcal{H}^{1}\left(R_{S}^{+}\right)+\mathcal{H}^{1}\left(R_{S}^{-}\right)=\mathcal{H}^{1}\left(R_{S}^{+}\right)+\mathcal{H}^{1}\left(Q R_{S}^{-}\right)=\mathcal{H}^{1}\left(G_{\nu}\right)
$$

The statement (2) immediately follows from Proposition 3.1(3). Eventually, let us prove $(3)$.

If $\Sigma^{*}$ is a rectifiable indecomposable factor of $\Sigma$ then, by [8, Theorem 4.1], we can find a couple of disjointed $\mathcal{H}^{1}$-measurable sets

$$
G^{\prime}, G^{\prime \prime} \subset \operatorname{spt} \Sigma^{*} \subset \operatorname{spt} \Sigma
$$

(the last inclusion being true by Proposition 4.2) such that $q G^{\prime}$ and $q G^{\prime \prime}$ are semicircles and

$$
q G^{\prime} \cap q G^{\prime \prime}=\emptyset \quad \text { or } \quad q G^{\prime}=q G^{\prime \prime}
$$


The area formula implies that

$$
\begin{aligned}
& \mathcal{H}^{1}\left(\operatorname{spt} \Sigma \backslash R_{S}\right) \geq \mathcal{H}^{1}\left(\left(G^{\prime} \cup G^{\prime \prime}\right) \backslash R_{S}\right)=\mathcal{H}^{1}\left(G^{\prime} \backslash R_{S}\right)+\mathcal{H}^{1}\left(G^{\prime \prime} \backslash R_{S}\right) \\
\geq & \mathcal{H}^{1}\left(q\left(G^{\prime} \backslash R_{S}\right)\right)+\mathcal{H}^{1}\left(q\left(G^{\prime \prime} \backslash R_{S}\right)\right) \\
= & \mathcal{H}^{1}\left(q\left(G^{\prime} \backslash\left(R_{S} \cap G^{\prime}\right)\right)\right)+\mathcal{H}^{1}\left(q\left(G^{\prime \prime} \backslash\left(R_{S} \cap G^{\prime \prime}\right)\right)\right) \\
\geq & \mathcal{H}^{1}\left(q G^{\prime} \backslash q\left(R_{S} \cap G^{\prime}\right)\right)+\mathcal{H}^{1}\left(q G^{\prime \prime} \backslash q\left(R_{S} \cap G^{\prime \prime}\right)\right) \\
= & 2 \pi-\mathcal{H}^{1}\left(q\left(R_{S} \cap G^{\prime}\right)\right)-\mathcal{H}^{1}\left(q\left(R_{S} \cap G^{\prime \prime}\right)\right) \\
\geq & 2 \pi-\mathcal{H}^{1}\left(q\left(R_{S}^{+} \cap G^{\prime}\right)\right)-\mathcal{H}^{1}\left(q\left(R_{S}^{-} \cap G^{\prime}\right)\right) \\
& -\mathcal{H}^{1}\left(q\left(R_{S}^{+} \cap G^{\prime \prime}\right)\right)-\mathcal{H}^{1}\left(q\left(R_{S}^{-} \cap G^{\prime \prime}\right)\right) .
\end{aligned}
$$

As $q_{\mid R_{S}^{+}}$and $q_{\mid R_{S}^{-}}$are injective (being $M$ convex), one has also

$$
\mathcal{H}^{1}\left(q\left(R_{S}^{+} \cap G^{\prime}\right)\right)+\mathcal{H}^{1}\left(q\left(R_{S}^{+} \cap G^{\prime \prime}\right)\right)=\mathcal{H}^{1}\left(q\left(R_{S}^{+} \cap\left(G^{\prime} \cup G^{\prime \prime}\right)\right)\right) \leq \mathcal{H}^{1}\left(q R_{S}^{+}\right)
$$

and

$$
\mathcal{H}^{1}\left(q\left(R_{S}^{-} \cap G^{\prime}\right)\right)+\mathcal{H}^{1}\left(q\left(R_{S}^{-} \cap G^{\prime \prime}\right)\right)=\mathcal{H}^{1}\left(q\left(R_{S}^{-} \cap\left(G^{\prime} \cup G^{\prime \prime}\right)\right)\right) \leq \mathcal{H}^{1}\left(q R_{S}^{-}\right) .
$$

Hence we get

$$
\begin{aligned}
\mathcal{H}^{1}\left(\operatorname{spt} \Sigma \backslash R_{S}\right) & \geq 2 \pi-\mathcal{H}^{1}\left(q R_{S}^{+}\right)-\mathcal{H}^{1}\left(q R_{S}^{-}\right) \\
& =2 \pi-\mathcal{H}^{1}\left(q R_{S}^{+}\right)-\mathcal{H}^{1}\left(-q R_{S}^{-}\right) \\
& =2 \pi-\mathcal{H}^{1}\left(\nu\left(M_{S}^{+}\right)\right)-\mathcal{H}^{1}\left(\nu\left(M_{S}^{-}\right)\right) \\
& =2 \pi-\mathcal{H}^{1}(\nu(M))=\mathcal{H}^{1}\left(\tilde{G}_{\nu} \backslash G_{\nu}\right)
\end{aligned}
$$

i.e., just the thesis.

The following result is a corollary of Proposition 7.1.

Proposition 7.2. Let $M$ be convex. Then:

(1) The natural Gauss graphs of $M$ are minimizers;

(2) every minimizer is indecomposable.

Proof. Let $T:=\llbracket G, \eta, \theta \rrbracket$ be a minimizer.

First step. Consider a nonzero indecomposable factor $\Sigma$ of $T$. Then, by Proposition 4.3, one has

$$
\Sigma \in \operatorname{curv}_{1}\left(\mathbf{R}^{2}\right) \quad \text { and } \quad \text { spt } \Sigma \subset G .
$$

By Proposition 7.1 we have:

$$
\begin{aligned}
\mathbf{M}(T) \leq \mathcal{H}^{1}\left(\tilde{G}_{\nu}\right) & =\mathcal{H}^{1}\left(\tilde{G}_{\nu} \backslash G_{\nu}\right)+\mathcal{H}^{1}\left(G_{\nu}\right) \leq \mathcal{H}^{1}\left(\operatorname{spt} \Sigma \backslash R_{G}\right)+\mathcal{H}^{1}\left(R_{G}\right) \\
& =\mathcal{H}^{1}\left(\operatorname{spt} \Sigma \cup R_{G}\right) \leq \mathcal{H}^{1}(G) \leq \int_{G} \theta d \mathcal{H}^{1}=\mathbf{M}(T)
\end{aligned}
$$


whence we get the first part of the thesis and also that

$$
\theta \equiv 1
$$

and

$$
\text { spt } \Sigma \cup R_{G}=G \text {. }
$$

Second step. By $[11,4.2 .25]$ and recalling that every null-boundary, nonzero, one-dimensional generalized Gauss graph has mass not smaller than $2 \pi$ (see [8, Theorem 4.1]), one can find a finite proper decomposition of $T$ whose factors are indecomposable currents in $I_{1}\left(\mathbf{R}_{x}^{2} \times \mathbf{R}_{y}^{2}\right)$ : let them be denoted by $\Sigma_{j}:=\llbracket G_{j}, \eta_{j}, 1 \rrbracket(j=1, \ldots, h)$.

Now assume $h \geq 2$ : we shall get a contradiction.

By (7.1) and Proposition 4.2 one has that

$$
G=\cup_{j=1}^{h} G_{j} \quad \text { and } \quad G_{i} \cap G_{j}=\emptyset \text { whenever } i \neq j .
$$

Moreover, by (7.2), we know that

$$
G_{j} \cup R_{G}=G
$$

for $j=1, \ldots, h$. It follows that $G_{j} \subset R_{G}$ for $j=1, \ldots, h$ and hence $G \subset R_{G}$. The opposite inclusion holds by Proposition 3.1(3), whereby we obtain

$$
R_{G}=G
$$

As $\partial T=0$ and $G$ has to be Lipschitz (see [11, 4.2.25]), we conclude that $G$ has no hole and thus

$$
R_{G}=G_{\nu} \quad \text { or } \quad R_{G}=Q G_{\nu} .
$$

We get

$$
G=\cup_{j=1}^{h} G_{j}=G_{\nu} \quad \text { or } \quad G=\cup_{j=1}^{h} G_{j}=Q G_{\nu}
$$

which cannot be true, being $h \geq 2$.

The following proposition improves the previous one.

Proposition 7.3. Let $M$ be convex. Then a generalized Gauss graph is a minimizer if and only if it is a natural Gauss graph of $M$. 
Proof. By Proposition 7.2 we have only to prove that a minimizer has to be a natural Gauss graph.

Let $\Sigma$ be a minimizer and $S:=$ spt $\Sigma$. Then Proposition 7.1 and Proposition 7.2 imply that

$$
\begin{aligned}
\mathcal{H}^{1}\left(S \backslash R_{S}\right) & =\mathcal{H}^{1}(S)-\mathcal{H}^{1}\left(R_{S}\right)=\mathcal{H}^{1}\left(\tilde{G}_{\nu}\right)-\mathcal{H}^{1}\left(G_{\nu}\right) \\
& =\mathcal{H}^{1}\left(\tilde{G}_{\nu} \backslash G_{\nu}\right)=\mathcal{H}^{1}\left(S_{1}\left(\mathbf{R}^{2}\right) \backslash \nu(M)\right) .
\end{aligned}
$$

Thus, by recalling the inequality obtained in the proof of Proposition 7.1, we get:

$$
\mathcal{H}^{1}\left(G^{\prime} \backslash R_{S}\right)+\mathcal{H}^{1}\left(G^{\prime \prime} \backslash R_{S}\right)=\mathcal{H}^{1}\left(q\left(G^{\prime} \backslash R_{S}\right)\right)+\mathcal{H}^{1}\left(q\left(G^{\prime \prime} \backslash R_{S}\right)\right)
$$

and

$$
\mathcal{H}^{1}\left(S \backslash R_{S}\right)=\mathcal{H}^{1}\left(\left(G^{\prime} \cup G^{\prime \prime}\right) \backslash R_{S}\right)
$$

whence it follows that

$$
\mathcal{H}^{1}\left(G^{\prime} \backslash R_{S}\right)=\mathcal{H}^{1}\left(q\left(G^{\prime} \backslash R_{S}\right)\right), \quad \mathcal{H}^{1}\left(G^{\prime \prime} \backslash R_{S}\right)=\mathcal{H}^{1}\left(q\left(G^{\prime \prime} \backslash R_{S}\right)\right)
$$

and

$$
S \backslash R_{S}=\left(G^{\prime} \cup G^{\prime \prime}\right) \backslash R_{S} .
$$

The first couple of equalities says that " $G^{\prime} \backslash R_{S}$ and $G^{\prime \prime} \backslash R_{S}$ are vertical", i.e., that

$$
\mathcal{H}^{1}\left(p\left(G^{\prime} \backslash R_{S}\right)\right)=\mathcal{H}^{1}\left(p\left(G^{\prime \prime} \backslash R_{S}\right)\right)=0
$$

whereby the last equality implies the "verticality" of $S \backslash R_{S}$ :

$$
\begin{aligned}
\mathcal{H}^{1}\left(p\left(S \backslash R_{S}\right)\right) & =\mathcal{H}^{1}\left(p\left(\left(G^{\prime} \cup G^{\prime \prime}\right) \backslash R_{S}\right)\right) \\
& =\mathcal{H}^{1}\left(p\left(G^{\prime} \backslash R_{S}\right) \cup p\left(G^{\prime \prime} \backslash R_{S}\right)\right) \\
& \leq \mathcal{H}^{1}\left(p\left(G^{\prime} \backslash R_{S}\right)\right)+\mathcal{H}^{1}\left(p\left(G^{\prime \prime} \backslash R_{S}\right)\right)=0 .
\end{aligned}
$$

Hence we get

$$
\mathcal{H}^{1}(p S)=\mathcal{H}^{1}\left(p\left(S \backslash R_{S}\right) \cup p R_{S}\right)=\mathcal{H}^{1}\left(p R_{S}\right)
$$

and thus

$$
p S=p R_{S}=M .
$$

The conclusion easily follows by recalling that $\mathrm{S}$ is Lipschitz.

\section{References}

[1] G. Anzellotti, Functionals depending on curvatures, Rend. Sem. Mat. Univ. Politec. Torino. Fascicolo speciale 1989: P.D.E. and Geometry, (1988), 47-62. 
[2] G. Anzellotti and S. Delladio, Minimization of Functionals of Curvatures and the Willmore Problem, Proceedings of the International Conference on Geometric Analysis and Continuum Mechanics, Stanford, 1993.

[3] G. Anzellotti, S. Delladio and G. Scianna, BV Functions over Rectifiable Currents, Annali Matem. Pura Appl., Firenze, CLXX (1996), 257-296.

[4] G. Anzellotti and E. Ossanna, Singular sets of surfaces with generalized curvatures, Manuscripta Math., 86 (1995), 417-433.

[5] G. Anzellotti, R. Serapioni and I. Tamanini, Curvatures, Functionals, Currents, Indiana Univ. Math. J., 39 (1990), 617-669.

[6] M. Berger and B. Gostiaux, Differential Geometry: Manifolds, Curves, and Surfaces, Springer-Verlag, New York, 1988.

[7] S. Delladio, Lower semicontinuity and continuity of functions of measures with respect to the strict convergence, The Royal Society of Edinburgh Proceedings, 119A (1991), 265-278.

[8] _ Slicing of Generalized Surfaces with Curvatures Measures and Diameter's Estimate, Annales Polonici Mathematici, LXIV.3 (1996), 267-283.

[9] Do Generalized Gauss Graphs Induce Curvature Varifolds, Bollettino Unione Matem. Italiana, 10-B (1996), 991-1017.

[10] S. Delladio and G. Scianna, Oriented and nonoriented curvature varifolds, The Royal Society of Edinburgh Proceedings, 125A (1995), 63-83.

[11] H. Federer, Geometric Measure Theory, Springer-Verlag, 1969.

[12] J.E. Hutchinson, Second fundamental form for varifolds and the existence of surfaces minimizing curvature, Indiana Univ. Math. J., 35 (1986), 45-71.

[13] $\quad C^{1, \alpha}$ multiple function and tangent cone behaviour for varifolds with second fundamental form in $L^{p}$, Proc. Sympos. Pure Math., 44 (1986), 281-306.

[14] Some regularity theory for curvature varifolds, Proc. Center Math. Analysis, Australian National University, 12 (1987), 60-66.

[15] C. Mantegazza, Curvature Varifolds with boundary, to appear on J. of Differential Geometry.

[16] M. Marcus, Finite Dimensional Multilinear Algebra, Part II, Marcel Dekker Inc., New York, 1975.

[17] F. Morgan, Geometric Measure Theory, a beginner's guide, Academic Press Inc., 1988.

[18] U. Pinkall and I. Sterling, Willmore surfaces, Math. Intelligencer, 9 (1987), 38-43.

[19] W. Rudin, Real and Complex Analysis, McGraw-Hill, 1970.

[20] L. Simon, Lectures on Geometric Measure Theory, Proceedings of the Centre for Mathematical Analysis, Canberra, Australia, 3 (1984).

[21] Existence of Willmore Surfaces, Proc. Centre for Math. Anal., 10 (1985), $187-216$.

[22] Existence of surfaces minimizing the Willmore functional, Comm. Anal. Geom., 2 (1993), 281-326.

[23] T.J. Willmore, Note on embedded surfaces, An. Stiint. Univ. "Al. I. Cusa" Iasi Sect. I, a Mat., 11B (1965), 493-496. 
[24] - Mean curvature of immersed surfaces, An. Stiint. Univ. "Al. I. Cusa" Iasi Sect. I, a Mat., 14 (1968), 99-103.

Received December 21, 1995.

UNIVERSITÀ DI TRENTO

38050 Povo

TRENTO, ITALY

E-mail address: delladio@science.unitn.it 